\title{
Brain Drain: The Mere Presence of One's Own Smartphone Reduces Available Cognitive Capacity
}

\author{
ADRIAN F. WARD, KRISTEN DUKE, AYELET GNEEZY, AND MAARTEN W. BOS
}

\begin{abstract}
Our smartphones enable - and encourage - constant connection to information, entertainment, and each other. They put the world at our fingertips, and rarely leave our sides. Although these devices have immense potential to improve welfare, their persistent presence may come at a cognitive cost. In this research, we test the "brain drain" hypothesis that the mere presence of one's own smartphone may occupy limited-capacity cognitive resources, thereby leaving fewer resources available for other tasks and undercutting cognitive performance. Results from two experiments indicate that even when people are successful at maintaining sustained attention —as when avoiding the temptation to check their phones - the mere presence of these devices reduces available cognitive capacity. Moreover, these cognitive costs are highest for those highest in smartphone dependence. We conclude by discussing the practical implications of this smartphone-induced brain drain for consumer decision-making and consumer welfare.
\end{abstract}

We all understand the joys of our always-wired world — the connections, the validations, the laughs ... the info. ... But we are only beginning to get our minds around the costs.

_-Andrew Sullivan (2016)

$\bar{T}$ he proliferation of smartphones has ushered in an era of unprecedented connectivity. Consumers around the globe are now constantly connected to faraway friends, endless entertainment, and virtually unlimited information. With smartphones in hand, they check the weather from bed, trade stocks - and gossip — while stuck in traffic, browse potential romantic partners between appointments, make online purchases while standing in-store, and live-stream each others' experiences, in real time, from opposite sides of the globe. Just a decade ago, this state of constant connection would have been inconceivable; today, it is seemingly indispensable. ${ }^{1}$ Smartphone owners interact with their phones an average of 85 times a day, including immediately upon waking up, just before going to sleep, and even in the middle of the night (Perlow 2012; Andrews et al. 2015; dscout 2016). Ninety-one percent report that they never leave home without their phones (Deutsche Telekom 2012), and 46\% say that they couldn't live without them (Pew Research Center 2015). These revolutionary devices enable on-demand access to friends, family, colleagues, companies, brands, retailers, cat videos, and much more. They represent all that the connected world has to offer, condensed into a device that fits in the palm of one's hand-and almost never leaves one's side.

The sharp penetration of smartphones, both across global markets and into consumers' everyday lives, represents a phenomenon high in "meaning and mattering" (e.g., Kernan 1979; Mick 2006) —one that has the potential to affect the welfare of billions of consumers worldwide. As individuals increasingly turn to smartphone screens for managing and enhancing their daily lives, we must ask how dependence on these devices affects the ability to

Adrian F. Ward (adrian.ward@mccombs.utexas.edu) is an assistant professor of marketing in the McCombs School of Business, University of Texas at Austin, 2110 Speedway, Austin, TX 78712. Kristen Duke (kristen.duke@rady.ucsd.edu) is a PhD candidate in marketing at the Rady School of Management, University of California, San Diego, 9500 Gilman Drive, La Jolla, CA 92093. Ayelet Gneezy (agneezy@ucsd.edu) is an associate professor of behavioral sciences and marketing at the Rady School of Management, University of California, San Diego, 9500 Gilman Drive, La Jolla, CA 92093. Maarten W. Bos (mbos @disneyresearch.com) is a research scientist at Disney Research, 4720 Forbes Avenue, Pittsburgh, PA 15213. The authors thank Jiyoung Lee, Stephanie Schwartz, Yael Horwitz, and the Atkinson Behavioral Lab for research assistance.

1. In 2007, only $4 \%$ of American adults owned smartphones (Radwanick 2012). As of January 2017, 77\% of American adults—and $92 \%$ of those under the age of 35- own smartphones (Pew Research Center 2017). Penetration is similarly high in most Western nations, and even higher in several Middle Eastern and Asian countries. South Korea, for example, has a national smartphone ownership rate of 88\%, including $100 \%$ of those under 35 (Pew Research Center 2016).

JACR, volume 2, number 2. Published online April 3, 2017. http://dx.doi.org/10.1086/691462

(C) 2017 the Association for Consumer Research. All rights reserved. 2378-1815/2017/0202-0009\$10.00 
think and function in the world off-screen. Smartphones promise to create a surplus of resources, productivity, and time (e.g., Turkle 2011; Lee 2016); however, they may also create unexpected deficits. Prior research on the costs and benefits associated with smartphones has focused on how consumers' interactions with their smartphones can both facilitate and interrupt off-screen performance (e.g., Isikman et al. 2016; Sciandra and Inman 2016). In the present research, we focus on a previously unexplored (but common) situation: when smartphones are not in use, but are merely present.

We propose that the mere presence of one's own smartphone may induce "brain drain" by occupying limitedcapacity cognitive resources for purposes of attentional control. Because the same finite pool of attentional resources supports both attentional control and other cognitive processes, resources recruited to inhibit automatic attention to one's phone are made unavailable for other tasks, and performance on these tasks will suffer. We differentiate between the orientation and allocation of attention and argue that the mere presence of smartphones may reduce the availability of attentional resources even when consumers are successful at controlling the conscious orientation of attention.

\section{COGNITIVE CAPACITY AND CONSUMER BEHAVIOR}

Consumers' finite capacity for cognitive processing is one of the most fundamental influences on "real world" consumer behavior (e.g., Bettman 1979; Bettman, Johnson, and Payne 1991). Individuals are constantly surrounded by potentially meaningful information; however, their ability to use this information is consistently constrained by cognitive systems that are capable of attending to and processing only a small amount of the information available at any given time (e.g., Craik and Lockhart 1972; Newell and Simon 1972). This capacity limit shapes a wide range of behaviors, from in-the-moment decision-making strategies and performance (e.g., Lane 1982; Lynch and Srull 1982) to long-term goal pursuit and self-regulation (e.g., Hofmann, Strack, and Deutsch 2008; Benjamin, Brown, and Shapiro 2013).

Consumers' cognitive capabilities - and constraints - are largely determined by the availability of domain-general, limited-capacity attentional resources associated with both working memory and fluid intelligence (e.g., Halford, Cowan, and Andrews 2007; Jaeggi et al. 2008). "Working memory" (WM) refers to the theoretical cognitive system that sup- ports complex cognition by actively selecting, maintaining, and processing information relevant to current tasks and/ or goals. "Working memory capacity" (WMC) reflects the availability of attentional resources, which serve the "central executive" function of controlling and regulating cognitive processes across domains (Baddeley and Hitch 1974; Miyake and Shah 1999; Engle 2002; Baddeley 2003). "Fluid intelligence" (Gf) represents the ability to reason and solve novel problems, independent of any contributions from acquired skills and knowledge stored in "crystallized intelligence" (Cattell 1987). Similar to WM, Gf stresses the ability to select, store, and manipulate information in a goal-directed manner. Also similar to WM, Gf is constrained by the availability of attentional resources (e.g., Engle et al. 1999; Halford et al. 2007). Crucially, the limited capacity of these domaingeneral resources dictates that using attentional resources for one cognitive process or task leaves fewer available for other tasks; in other words, occupying cognitive resources reduces available cognitive capacity.

Given the chronic mismatch between the abundance of environmental information and the limited ability to process that information, individuals need to be selective in their allocation of attentional resources (e.g., Kahneman 1973; Johnston and Dark 1986). The priority of a stimulus - that is, the likelihood that it will attract attentionis determined by both its physical "salience" (e.g., location, perceptual contrast) and its goal "relevance" (i.e., potential importance for goal-directed behavior) (e.g., Corbetta and Shulman 2002; Fecteau and Munoz 2006). Preferential attention to temporarily relevant stimuli, such as those associated with a current task or decision, is supported by WM; when a goal is active in WM, stimuli relevant to that goal are more likely to attract attention (e.g., Moskowitz 2002; Soto et al. 2005; Vogt et al. 2010). Frequently relevant stimuli, such as those associated with long-term and/or selfrelevant goals, may automatically attract attention even when the goals associated with these stimuli are not active in WM (Shiffrin and Schneider 1977; Johnston and Dark 1986); for example, individuals automatically orient to the sounds of their own names in ignored audio channels (Moray 1959), and mothers, more so than nonmothers, automatically attend to infants' emotional expressions (Thompson-Booth et al. 2014). Automatic attention generally helps individuals make the most of their limited cognitive capacity by directing attention to frequently goalrelevant stimuli without requiring these goals to be constantly kept in mind. However, automatic attention may undermine performance when an environmental stimulus is 
frequently relevant to an individual's goals but currently irrelevant to the task at hand; inhibiting automatic attentionkeeping attractive but task-irrelevant stimuli from interfering with the contents of consciousness - occupies attentional resources (e.g., Engle 2002).

Smartphones serve as consumers' personal access points to all the connected world has to offer. We suggest that the increasing integration of these devices into the minutiae of daily life both reflects and creates a sense that they are frequently relevant to their owners' goals; it lays the foundation for automatic attention. Consistent with this position, research indicates that signals from one's own phone (but not someone else's) activate the same involuntary attention system that responds to the sound of one's own name (Roye, Jacobsen, and Schröger 2007). When these devices are salient in the environment, their status as high-priority (relevant and salient) stimuli suggests that they will exert a gravitational pull on the orientation of attention. And when consumers are engaged in tasks for which their smartphones are task-irrelevant, the ability of these devices to automatically attract attention may undermine performance in two ways (Clapp, Rubens, and Gazzaley 2009; Clapp and Gazzaley 2012). First, smartphones may redirect the orientation of conscious attention away from the focal task and toward thoughts or behaviors associated with one's phone. Prior research provides ample evidence that individuals spontaneously attend to their phones at inopportune times (e.g., Oulasvirta et al. 2011), and that this digital distraction adversely affects both performance (End et al. 2009) and enjoyment (Isikman et al. 2016). Second, smartphones may redistribute the allocation of attentional resources between engaging with the focal task and inhibiting attention to one's phone. Because inhibiting automatic attention occupies attentional resources, performance on tasks that rely on these resources may suffer even when consumers do not consciously attend to their phones. We explore this possibility in the current research.

\section{SMARTPHONE USE AND CONSCIOUS DISTRACTION (THE ORIENTATION OF ATTENTION)}

Research on the relationship between mobile devices and cognitive functioning has largely focused on downstream consequences of device-related changes in the orientation of attention. For example, research on mobile device use while driving indicates that interacting with one's phone while behind the wheel causes performance deficits such as delayed reaction times and inattentional blindness (e.g.,
Strayer and Johnston 2001; Caird et al. 2008); these deficits mirror those associated with distracting "live" conversations (Recarte and Nunes 2003). Similarly, research in the educational sphere demonstrates that using mobile devices and social media while learning new material reduces comprehension and impairs academic performance (e.g., Froese et al. 2012). However, mobile device use does not affect performance on self-paced tasks, which allow individuals to compensate for device-related distractions by picking up where they left off (e.g., Fox, Rosen, and Crawford 2009; Bowman et al. 2010). Taken together, these findings suggest that many of the cognitive impairments associated with mobile device use may simply represent the general deleterious effects of diverting conscious attention away from a focal task. What may be special about smartphones, however, is the frequency with which they seem to create these diversions; their omnipresence and personal relevance may combine to create a particularly potent draw on the orientation of attention.

A more limited body of work explores the cognitive consequences of smartphone-related distractions in the absence of behavioral interaction (i.e., when consumers consciously think about phone-related stimuli, but do not actually use their phones). Research on the attentional cost of receiving cellphone notifications indicates that awareness of a missed text message or call impairs performance on tasks requiring sustained attention, arguably because unaddressed notifications prompt message-related (and task-unrelated) thoughts (Stothart, Mitchum, and Yehnert 2015). Related research shows that individuals who hear their phones ring while being separated from them report decreased enjoyment of focal tasks as a consequence of increased attention to phone-related thoughts (Isikman et al. 2016). Forced separation from one's ringing phone can also increase heart rate and anxiety and decrease cognitive performance (Clayton, Leshner, and Almond 2015). To our knowledge, only one prior study has investigated the cognitive effects of the mere presence of a mobile device - one that is not ringing, buzzing, or otherwise actively interfering with a focal task. Thornton et al. (2014, 485-86) found that a visually salient cellphone can impair performance on tasks requiring sustained attention by eliciting awareness of the "broad social and informational network . . . that one is not part of at the moment." Together, these investigations of phone-related distractions provide evidence that mobile devices can adversely affect cognitive performance even when consumers are not actively using them. Similar to earlier research on distracted driving and learning while multitasking, however, these studies 
connect the cognitive costs of smartphones to their (remarkable) ability to attract the conscious orientation of attention. When individuals interact with or think about their phones rather than attend to the task at hand, their performance suffers.

\section{SMARTPHONE PRESENCE AND COGNITIVE CAPACITY (THE ALLOCATION OF ATTENTIONAL RESOURCES)}

We suggest that smartphones may also impair cognitive performance by affecting the allocation of attentional resources, even when consumers successfully resist the urge to multitask, mind-wander, or otherwise (consciously) attend to their phones - that is, when their phones are merely present. Despite the frequency with which individuals use their smartphones, we note that these devices are quite often present but not in use-and that the attractiveness of these high-priority stimuli should predict not just their ability to capture the orientation of attention, but also the cognitive costs associated with inhibiting this automatic attention response.

We propose that the mere presence of one's smartphone may impose a "brain drain" as limited-capacity attentional resources are recruited to inhibit automatic attention to one's phone, and are thus unavailable for engaging with the task at hand. Research on controlled versus automatic processing provides evidence that the mere presence of personally relevant stimuli can impair performance on cognitive tasks (e.g., Geller and Shaver 1976; Bargh 1982; Wingenfeld et al. 2006). Importantly, these performance deficits occur without conscious attention to the potentially interfering stimuli and as a function of inhibiting these stimuli from interfering with the contents of consciousness (e.g., Shallice 1972; Lavie et al. 2004). Consistent with this evidence, we posit that the mere presence of consumers' own smartphones can reduce the availability of attentional resources (i.e., cognitive capacity) even when consumers are successful at controlling the conscious orientation of attention (i.e., resisting overt distraction).

If smartphones undermine cognitive performance by occupying attentional resources, the cognitive consequences of smartphone presence should be sensitive to variation in both the salience and the personal relevance of these devices, which together determine their priority in attracting attention (e.g., Fecteau and Munoz 2006). Prior research suggests that smartphones are chronically salient for many individuals, even when they are located out of sight in one's pocket or bag (e.g., Deb 2015). However, we expect that in- creasing the salience of one's smartphone-for example, by placing it nearby and in the field of vision - will amplify the cognitive costs associated with its presence, as more attentional resources are required to inhibit its influence on the orientation of attention. We also expect that these costs will vary according to the personal relevance of one's smartphone. We operationalize relevance in terms of "smartphone dependence," or the extent to which individuals rely on their phones in their everyday lives. We posit that individual differences in dependence on one's smartphone will moderate the effects of smartphone salience on available cognitive capacity, such that individuals who most depend on their phones will suffer the most from their presenceand benefit the most from their absence.

\section{OVERVIEW OF THE EXPERIMENTS}

In two experiments, we test the hypothesis that the mere presence of one's own smartphone reduces available cognitive capacity. We manipulate smartphone salience by asking participants to place their devices nearby and in sight (high salience, "desk" condition), nearby and out of sight (medium salience, "pocket/bag" condition), or in a separate room (low salience, "other room" condition). ${ }^{2}$ Our data indicate that the mere presence of one's smartphone adversely affects two domain-general measures of cognitive capacity - available working memory capacity (WMC) and functional fluid intelligence (Gf) - even when participants are not using their phones and do not report thinking about them (experiment 1). Data from experiment 2 replicate this effect on available cognitive capacity, show no effect on a behavioral measure of sustained attention, and provide evidence that individual differences in consumers' dependence on their devices moderate the effects of smartphone salience on available WMC.

\section{EXPERIMENT 1: SMARTPHONE SALIENCE AFFECTS AVAILABLE COGNITIVE CAPACITY}

In experiment 1 , we test the proposition that the mere presence of one's own smartphone reduces available cognitive capacity, as reflected in performance on tests of WMC

2. A pilot study confirmed that these physical locations predict individuals' top-of-mind awareness of their smartphones, with a nearby and in sight $\rightarrow$ nearby and out of sight $\rightarrow$ not nearby linear trend ( $F(1$, $111)=14.58, p<.001$, partial $\eta^{2}=.116$ ) and no quadratic trend ( $p=$ .996). Interestingly, the majority of respondents $(67.5 \%)$ indicated that they typically keep their smartphones nearby and in sight, where these devices are most salient. See the appendix for method and detailed analyses. 
and Gf. Each of these domain-general cognitive constructs is constrained by the availability of attentional resources, and the moment-to-moment availability of these resources predicts performance on tests of both WMC (Engle, Cantor, and Carullo 1992; Ilkowska and Engle 2010) and Gf (Horn 1972; Mani et al. 2013). If the mere presence of one's own smartphone taxes the limited-capacity attentional resources that constrain both WMC and Gf, then the salience of this device should predict performance on tasks associated with these constructs. We test this hypothesis in experiment 1 .

\section{Method}

Participants. Five hundred forty-eight undergraduates (53.3\% female; $M_{\text {age }}=21.1$ years; $\mathrm{SD}_{\text {age }}=2.4$ years) participated for course credit. Data collection spanned two weeks. Duplicate data from repeat participants were discarded prior to analysis. We applied the same three data selection criteria in experiments 1 and 2; see the appendix (available online) for additional detail. In experiment 1 , three participants were excluded for indicating they did not own smartphones, eight participants were excluded for failing to follow instructions, and seventeen participants were excluded due to excessive error rates on the OSpan task (less than 85\% accuracy; see Unsworth et al. 2005). Our final sample consisted of 520 smartphone users.

Procedure. We manipulated smartphone salience by randomly assigning participants to one of three phone location conditions: desk, pocket/bag, or other room. Participants in the "other room" condition left all of their belongings in the lobby before entering the testing room (as per typical lab protocol). Participants in the "desk" condition left most of their belongings in the lobby but took their phones into the testing room "for use in a later study;" once in the testing room, they were instructed to place their phones face down in a designated location on their desks. Participants in the "pocket/bag" condition carried all of their belongings into the testing room with them and kept their phones wherever they "naturally" would. Of the 174 participants in this condition, 91 (52.3\%) reported keeping their phones in their pockets, and $83(47.7 \%)$ reported keeping their phones in their bags; a planned contrast revealed no difference between these groups on our key dependent variable ( $p=.17$ ), and they were pooled for all subsequent analyses. Participants in all conditions were instructed to "turn your phones completely on silent; this means turn off the ring and vibrate so that your phone won't make any sounds."
After participants entered the testing room, they completed two tasks intended to measure available cognitive capacity: the Automated Operation Span task (OSpan; Unsworth et al. 2005) and a 10-item subset of Raven's Standard Progressive Matrices (RSPM; Raven, Raven, and Court 1998). The OSpan task, a prominent measure of WMC, assesses the ability to keep track of task-relevant information while engaging in complex cognitive tasks. This particular measure was designed to stress the domain-general nature of the attentional resources at the heart of the WM system (Turner and Engle 1989); in each trial set, participants complete a series of math problems (information processing) while simultaneously updating and remembering a randomly generated letter sequence (information maintenance). Performance on the OSpan assesses the domaingeneral attentional resources "available to the individual on a moment-to-moment basis" (Engle et al. 1992). The RSPM test, a nonverbal measure of Gf, was developed to isolate individuals' capacity for understanding and solving novel problems (fluid intelligence), independent of any influence of accumulated knowledge or domain-specific skill (crystallized intelligence). In each trial, participants are shown an incomplete pattern matrix and asked to select the element that best completes the pattern. Much like the OSpan task, performance on the RSPM test is sensitive to the current availability of attentional resources (e.g., Mani et al. 2013). Complete details of the tasks and measures used in experiments 1 and 2 are provided in the appendix.

Participants also completed an exploratory test of the "ending-digit drop-off" effect, modeled after the procedure of Bizer and Schindler (2005). In this task, participants are shown a series of products with .99-ending and .00-ending prices and asked to report the quantity they would be able to purchase for $\$ 73$. Overestimating purchasing power for a .99-ending price relative to a matched .00 -ending price (e.g., \$3.99 vs. \$4.00) constitutes evidence of the drop-off effect. We thought this effect might be more pronounced for those whose phones were made salient. However, we failed to replicate the basic effect and did not find any evidence of ending-digit drop-off in any condition ( $F(1$, $514)=.20, p=.65)$. See the appendix for detailed analyses and results.

Next, participants completed a questionnaire that included items related to their experiences in the lab and their lay beliefs about the connection between smartphones and performance. These questions assessed how often they thought about their phones during the experiment, to what extent they thought the locations of their phones affected 
their performance in the lab, how they thought phone location might have affected their performance, and to what extent they believed their phones affected their performance and attention spans more generally; all responses were measured using 7-point Likert scales. Finally, participants answered a series of demographic questions (gender, age, ethnicity, nationality) and provided information about their cellphone make/model and data plan.

\section{Results and Discussion}

All analyses in experiment 1 include a "Week" factor to account for variation across research assistants; this factor does not interact with Phone Location in any analysis (all $F<1.27$, all $p>$.28).

Cognitive Capacity. We assessed the effects of smartphone salience on available cognitive capacity using two measures of domain-general cognitive function: OSpan task performance and RSPM test score. Because both tasks rely on limited-capacity attentional resources, both should be sensitive to fluctuations in the availability of these resources.

A multivariate analysis of variance (MANOVA) testing the effects of Phone Location (desk, pocket/bag, other room) on the optimal linear combination of these measures revealed a significant effect of Phone Location on cognitive

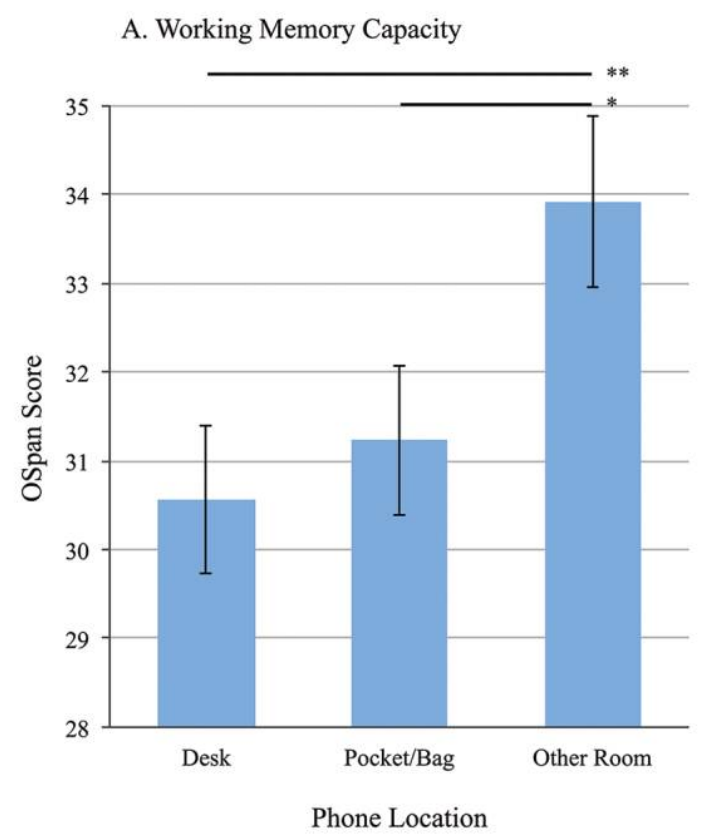

capacity (Pillai's Trace $=.027, F(4,1028)=3.51, p=.007$, partial $\left.\eta^{2}=.014\right)$. Paired comparisons revealed that participants in the "other room" condition performed better than those in the "desk" condition $(p=.002)$. Participants in the "pocket/bag" condition did not perform significantly differently from those in either the "desk" $(p=.09)$ or "other room" ( $p=.11$ ) conditions. However, planned contrasts revealed a significant desk $\rightarrow$ pocket/bag $\rightarrow$ other room linear trend (Pillai's Trace $=.023, F(2,513)=6.07, p=.002$, partial $\eta^{2}=.023$ ) and no quadratic trend (Pillai's Trace $=$ $.004, F(2,513)=.96, p=.39)$, suggesting that as smartphone salience increases, available cognitive capacity decreases.

Follow-up univariate ANOVAs separately testing the effect of Phone Location on OSpan performance and RSPM score were consistent with our focal multivariate analysis. Phone Location significantly affected both OSpan performance $\left(F(2,514)=3.74, p=.02\right.$, partial $\left.\eta^{2}=.014\right)$ and RSPM score $\left(F(2,514)=3.96, p=.02\right.$, partial $\eta^{2}=$ .015). See figure 1 for means, and the appendix for detailed analyses and results.

Conscious Thought. A one-way ANOVA on participants' responses to the question "While completing today's tasks, how often were you thinking about your cellphone?" (1 =

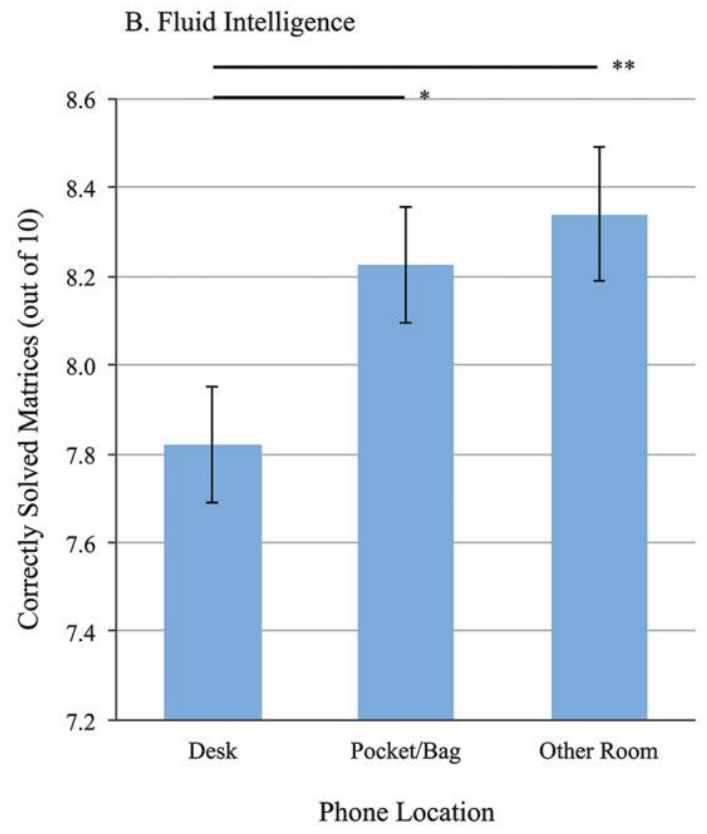

Figure 1. Experiment 1: effect of randomly assigned phone location condition on available WMC (OSpan Score, panel A) and functional Gf (Correctly Solved Raven's Matrices, panel B). Participants in the "desk" condition (high salience) displayed the lowest available cognitive capacity; those in the "other room" condition (low salience) displayed the highest available cognitive capacity. Error bars represent standard errors of the means. Asterisks indicate significant differences between conditions, with ${ }^{*} p<.05$ and ${ }^{* *} p<.01$. 
not at all to $7=$ constantly/the whole time) revealed no effect of Phone Location on phone-related thoughts ( $F(2$, $514)=.84, p=.43)$. Notably, the modal self-reported frequency of thinking about one's phone in each condition was "not at all." Combined with the significant effect of Phone Location on available cognitive capacity, these results support our proposition that the mere presence of one's smartphone may impair cognitive functioning even when it does not occupy the contents of consciousness.

Perceived Influence of Smartphone Presence. There were no differences between conditions on any measures related to the perceived effects of smartphones on performance ("How much / in what way do you think the position of your cellphone affected your performance on today's tasks?"; "In general, how much do you think your cellphone usually affects your performance and attention span?"), either in the context of the experiment (all $F<1.58$, all $p>.21$ ) or in general $(F(2,494)=2.26, p=.11)$. Across conditions, a majority of participants indicated that the location of their phones during the experiment did not affect their performance ("not at all"; 75.9\%) and "neither helped nor hurt [their] performance" (85.6\%). This contrast between perceived influence and actual performance suggests that participants failed to anticipate or acknowledge the cognitive consequences associated with the mere presence of their phones.

Discussion. The results of experiment 1 indicate that the mere presence of participants' own smartphones impaired their performance on tasks that are sensitive to the availability of limited-capacity attentional resources. In contrast to prior research, participants in our experiment did not interact with or receive notifications from their phones. In addition, self-reported frequency of thoughts about these devices did not differ across conditions. Taken together, these results suggest that the mere presence of one's smartphone may reduce available cognitive capacity and impair cognitive functioning, even when consumers are successful at remaining focused on the task at hand.

\section{EXPERIMENT 2: SMARTPHONE DEPENDENCE MODERATES THE EFFECT OF SMARTPHONE SALIENCE ON COGNITIVE CAPACITY}

The results of experiment 1 support the proposition that the mere presence of one's smartphone reduces available cognitive capacity, even when it is not in use. In experiment 2 , we replicate the basic design of experiment 1 , with the following exceptions. First, we conduct a stronger test of the proposed impairment-without-interruption effect by examining the effects of smartphone salience on both cognitive capacity (WMC) and a behavioral measure of sustained attention. Consistent with both the proposed theoretical framework and participants' self-reports in experiment 1 , we predict that increasing smartphone salience will adversely affect the availability of attentional resources without interrupting sustained attention. Second, one could argue that participants who had access to their phones in experiment 1 surreptitiously checked for notifications, were consciously distracted by unanswerable messages, and displayed impaired performance as a result (as in Clayton et al. 2015; Stothart et al. 2015; Isikman et al. 2016). We did not observe any behavior or this sort, and did not find any differences between conditions in the frequency of phonerelated thoughts. In experiment 2, we further address this alternate explanation by randomly assigning participants to either silence their phones (as in experiment 1 ) or turn them off completely. We predict that the salience of participants' smartphones will influence available cognitive capacity even when these devices are turned off and will not influence sustained attention even when they are turned on. Third, we test a potential moderator of the effects of smartphone salience on available cognitive capacity: individual differences in the personal relevance of one's phone, operationalized in terms of "smartphone dependence." We predict that individuals who are more dependent on their phones will be more affected by their presence.

\section{Method}

Participants. Two hundred and ninety-six undergraduates (56.9\% female; $M_{\text {age }}=21.3$ years; $\mathrm{SD}_{\text {age }}=2.6$ years) participated for course credit. Eleven participants were excluded for reporting that they did not own smartphones, four participants were excluded due to excessive error rates $(<85 \%$ OSpan accuracy), and six participants were excluded due to missing (5) or extreme (1) response times in a Go/No-Go task (see below). Our final sample consisted of 275 participants.

Procedure. This experiment followed a 3 (Phone Location: desk, pocket/bag, other room) $\times 2$ (Phone Power: on, off) between-subjects design. Phone Location instructions and randomization procedures were identical to those used in experiment 1, with the exception that participants in the "desk" condition were instructed to place their phones facing up. Of the 91 participants in the "pocket/bag" condition, $68(74.7 \%)$ reported keeping their phones in their 
pockets, and 23 (25.3\%) reported keeping their phones in their bags; as in experiment 1 , a planned contrast revealed no difference between these groups on our key dependent variable $(p=.55)$, and they were pooled for all subsequent analyses. Participants were randomly assigned to one of two Phone Power conditions prior to entering the testing room. The instructions for participants in the "power on" condition were identical to those used in experiment 1 ; we instructed participants in the "power off" condition to completely turn off their devices.

After placing their phones in the proper location and power mode, participants completed our two key dependent measures: the OSpan task and the Cue-Dependent Go/No-Go task (order counterbalanced across participants). In the Go/No-Go task, participants are presented with a series of "go" and "no go" targets, and instructed to respond to "go" targets as quickly as possible without making errors, but to refrain from responding to "no go" targets. In this task, both omission errors (failure to respond to "go" targets) and reaction time (speed of responding to these targets) serve as measures of sustained attention (Bezdjian et al. 2009). After completing both tasks, participants reported the subjective difficulty of each task.

Next, they completed a battery of exploratory questions intended to assess individual differences in use of and connection to one's smartphone, including a 13-item inventory related to reliance on one's phone (see appendix for all items and analyses). Finally, participants answered a series of demographic questions (gender, age, ethnicity, nationality) and provided information about their cellphone make/ model and data plan.

\section{Results and Discussion}

All analyses in experiment 2 include a Task Order reflecting our counterbalanced experimental design; this factor does not interact with Phone Location or Phone Location $\times$ Phone Power in any analysis (all $F<1.51$, all $p>.22$ ).

Cognitive Capacity. As in experiment 1, performance on the OSpan task measures the attentional resources available to the individual on a moment-to-moment basis (Engle et al. 1992). A 3 (Phone Location: desk, pocket/bag, other room) $\times 2$ (Phone Power: on, off) between-subjects ANOVA revealed a significant effect of Location on OSpan performance $\left(F(2,263)=3.53, p=.03\right.$, partial $\left.\eta^{2}=.026\right)$. There was no effect of Power $(F(1,263)=.05, p=.83)$ or of the Power $\times$ Location interaction $(F(2,263)=1.05, p=.35)$. Paired comparisons revealed that participants in the "other room" condition performed significantly better on the OSpan task than did those in the "desk" condition $\left(M_{\text {diff }}=4.67, p=\right.$ .008). Participants in the "pocket/bag" condition did not perform significantly differently from those in either the "desk" $\left(M_{\text {diff }}=2.30, p=.20\right)$ or "other room" $\left(M_{\text {diff }}=2.37, p=.17\right)$ conditions. See figure 2 for means.

Planned contrasts revealed a significant desk $\rightarrow$ pocket/ bag $\rightarrow$ other room linear trend $(F(1,263)=7.05, p=.008$, partial $\left.\eta^{2}=.026\right)$ and no quadratic trend $(F(1,263)=.001$, $p=.98)$. Consistent with experiment 1 , this pattern of results indicates that increasing the salience of one's smartphone impairs OSpan performance, and decreasing the salience of one's smartphone improves performance. Further, the null effects of Power and the Power $\times$ Location interaction suggest that decreases in performance are not related to incoming notifications (or the possibility of receiving notifications), ruling out this alternative explanation of the effects found in experiment 1.

Moderation by Smartphone Dependence. Our framework suggests that the effects of smartphone salience on available cognitive capacity should be moderated by individual differences in dependence on these devices. We tested this prediction by investigating responses to an exploratory 13-item inventory of individual differences in reliance on one's phone. A principal components factor analysis with Varimax rotation revealed that these items loaded onto two distinct factors, together explaining $52.67 \%$ of the variance. ${ }^{3}$ Factor 1 (Smartphone Dependence; six items) explained $31.02 \%$ of the variance and captured our primary concept of interest: the degree of dependence on one's smartphone (e.g., "I would have trouble getting through a normal day without my cellphone"). Factor 2 (Emotional Attachment; five items) explained $21.65 \%$ of the variance and accounted for the emotional aspects of smartphone use (e.g., "Using my cellphone makes me feel happy"). Reliability analyses indicated high reliability for both Smartphone Dependence $(\alpha=.89)$ and Emotional Attachment $(\alpha=.79)$ as distinct factors. See appendix table 1 for all items and factor loadings.

We tested the potential moderating role of Smartphone Dependence in a univariate generalized linear model predicting OSpan performance from all variables included in our original 3 (Phone Location: desk, pocket/bag, other room)

3. Two items did not clearly load onto either primary factor and were excluded from further analyses (Costello and Osborne 2005). 


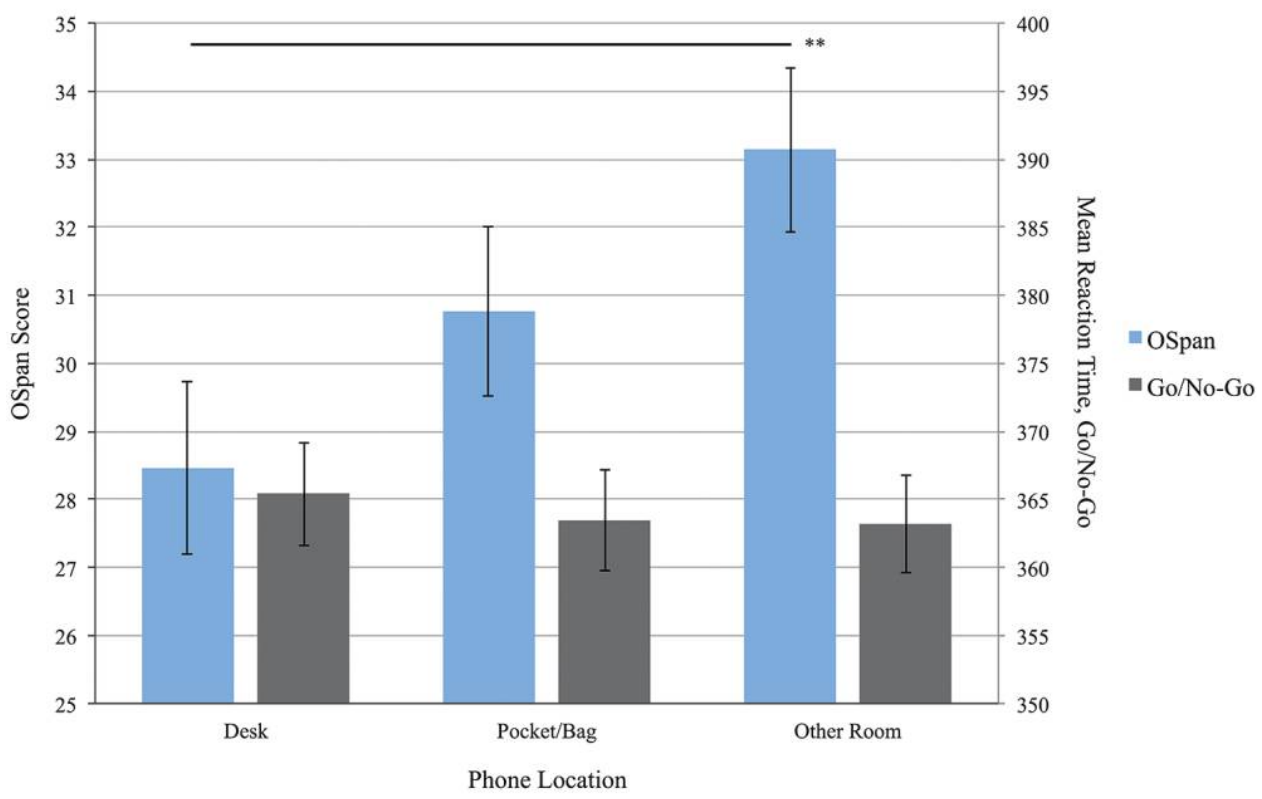

Figure 2. Experiment 2: effect of randomly assigned phone location condition on available cognitive capacity (OSpan Score) and sustained attention (Mean Reaction Time, Go/No-Go). Participants in the "desk" condition (high salience) displayed the lowest available cognitive capacity; those in the "other room" condition (low salience) displayed the highest available cognitive capacity. Phone location did not affect sustained attention. Error bars represent standard errors of the means. Asterisks indicate significant differences between conditions, with ${ }^{* *} p<.01$.

$\times 2$ (Phone Power: on, off) ANOVA, mean-centered Smartphone Dependence score, and all independent variable $\times$ Smartphone Dependence interaction terms (Baron and Kenny 1986). This analysis revealed a significant Phone Location $x$ Smartphone Dependence interaction $(F(2,247)=3.25$, $p=.04$, partial $\left.\eta^{2}=.026\right)$, indicating that the effects of smartphone salience on OSpan performance are moderated by individual differences in dependence on one's smartphone. Follow-up analyses probing the conditional effects of Location at the sample mean of the moderator and plus/ minus one SD from the mean revealed no effect of Location on OSpan performance at low levels of Smartphone Dependence $(-1 \mathrm{SD} ; p=.28)$; however, this effect was significant at both mean $(p=.05)$ and high levels $(p=.007)$ of Dependence. See figure 3 for estimated marginal means. Similar results for other measures of smartphone dependence (e.g., number of texts sent per day) are reported in the appendix.

Interestingly, a parallel moderation analysis indicated that Emotional Attachment did not moderate the effects of Phone Location on OSpan performance $(p=.61)$. Although we are cautious about making strong claims based on null effects and reiterate that these factors were derived from an exploratory inventory, this disparity between Smartphone Dependence and Emotional Attachment suggests that the effects of smartphone salience on available cognitive ca- pacity may be determined by the extent to which consumers feel they need their phones, as opposed to how much they like them. These results are consistent with the proposition that the effects of smartphone salience on available cognitive capacity stem from the singularly important role these devices play in many consumers' lives.

Sustained Attention. We analyzed the effects of smartphone salience on two behavioral measures of sustained attention: omission errors and reaction time in the Go/ No-Go task. A 3 (Phone Location: desk, pocket/bag, other room) $\times 2$ (Phone Power: on, off) ANOVA revealed no effects of Location, Power, or their interaction on either of these measures (all $F<1.05$, all $p>$.35). See figure 2 for reaction time means, and the appendix for full results.

Perceived Difficulty. Finally, we analyzed perceived task difficulty in order to see if the cognitive consequences of smartphone salience were reflected in participants' subjective experiences. A 3 (Phone Location: desk, pocket/bag, other room) $\times 2$ (Phone Power: on, off) ANOVA revealed a marginal effect of Location on perceived difficulty for the memory section of the OSpan task $(F(1,256)=2.38$, $p=.09$, partial $\left.\eta^{2}=.018\right)$. Paired comparisons revealed that participants in the "other room" condition found it sig- 


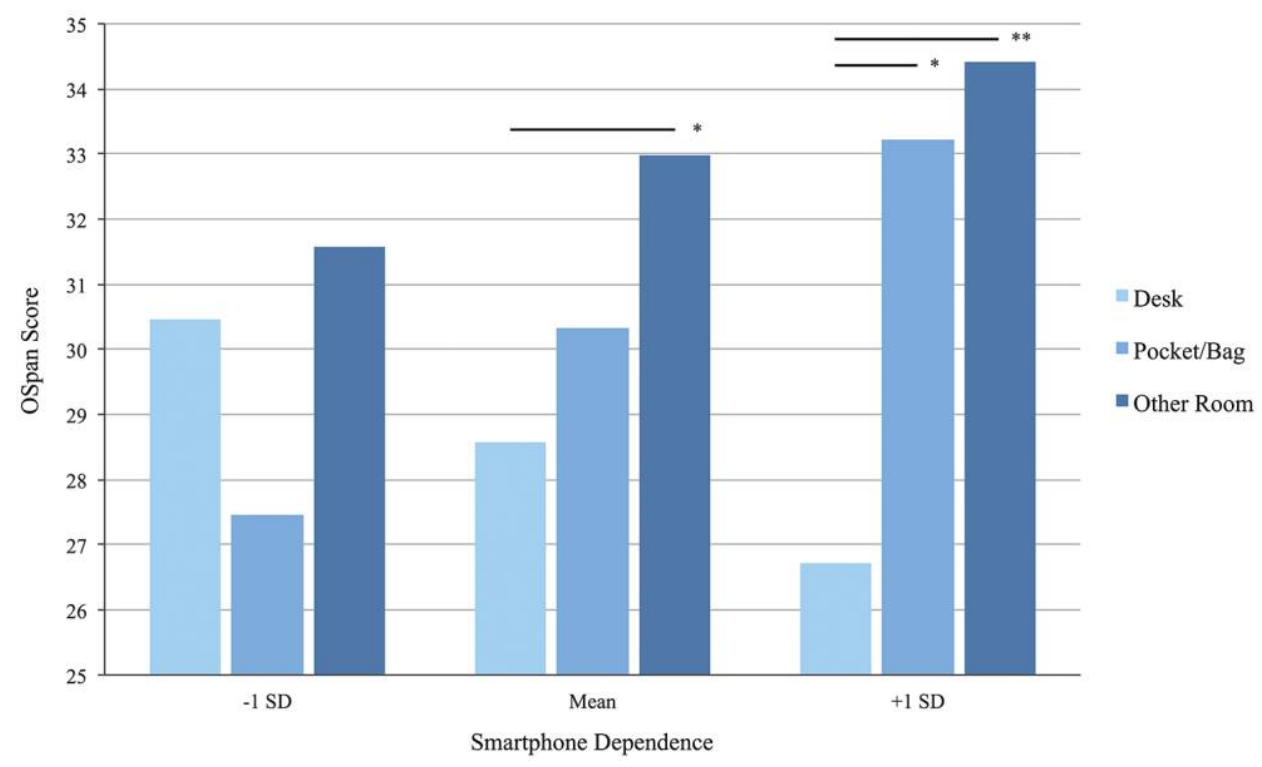

Figure 3. Experiment 2: estimated marginal means representing the effect of phone location on available cognitive capacity (OSpan Score) at low ( -1 SD), mean, and high (+1 SD) levels of smartphone dependence. Phone location affects available cognitive capacity at mean and high levels of smartphone dependence, but not at low levels of smartphone dependence. Asterisks indicate significant differences between conditions, with ${ }^{*} p<.05$ and ${ }^{* *} p<.01$.

nificantly easier to remember information in this task relative to participants in the "desk" condition $\left(M_{\text {diff }}=.49\right.$, $p=.04$ ) and marginally easier relative to those in the "pocket/bag" condition $\left(M_{\text {diff }}=.40, p=.09\right)$. This pattern of results is consistent with participants' actual performance on the OSpan task and suggests that the cognitive benefits of escaping the mere presence of one's phone may be reflected, at least partially, in subjective experience. However, the lay beliefs reported in experiment 1 suggest that even when consumers notice these benefits, they may not attribute them to the presence (or absence) of their phones. There were no differences between conditions on any of the other perceived difficulty or perceived performance measures (all $F<1.82$, all $p>$.16).

Discussion. Consistent with the behavioral and self-report results observed in experiment 1 , the results of experiment 2 suggest that the mere presence of consumers' own smartphones may adversely affect cognitive functioning even when consumers are not consciously attending to them. Experiment 2 also provides evidence that these cognitive costs are moderated by individual differences in dependence on these devices. Ironically, the more consumers depend on their smartphones, the more they seem to suffer from their presence - or, more optimistically, the more they may stand to benefit from their absence.

\section{GENERAL DISCUSSION}

The proliferation of smartphones represents a profound shift in the relationship between consumers and technology. Across human history, the vast majority of innovations have occupied a defined space in consumers' lives; they have been constrained by the functions they perform and the locations they inhabit. Smartphones transcend these limitations. They are consumers' constant companions, offering unprecedented connection to information, entertainment, and each other. They play an integral role in the lives of billions of consumers worldwide and, as a result, have vast potential to influence consumer welfareboth for better and for worse.

The present research identifies a potentially costly side effect of the integration of smartphones into daily life: smartphone-induced "brain drain." We provide evidence that the mere presence of consumers' smartphones can adversely affect two measures of cognitive capacity - available working memory capacity and functional fluid intelligencewithout interrupting sustained attention or increasing the frequency of phone-related thoughts. Consumers who were engaged with ongoing cognitive tasks were able to keep their phones not just out of their hands, but also out of their (conscious) minds; however, the mere presence of these devices left fewer attentional resources available for engaging with the task at hand. 
Further, we find that the effects of smartphone salience on available cognitive capacity are moderated by individual differences in the personal relevance of these devices (operationalized in terms of smartphone dependence); those who depend most on their devices suffer the most from their salience, and benefit the most from their absence. The role of dependence in determining mere presence effects suggests that similar cognitive costs would not be incurred by the presence of just any product, device, or even phone. We submit that few, if any, stimuli are both so personally relevant and so perpetually present as consumers' own smartphones. However, we leave open the door for our insights to apply more broadly to future connective technologies that may become equally central to consumers' lives as technology continues to advance.

Our research also offers insight into the tactics that might mitigate "brain drain" — as well as those that might not. For example, we find that the effect of smartphone salience on cognitive capacity is robust to both the visibility of the phone's screen (face down in experiment 1 , face up in experiment 2) and the phone's power (silent vs. powered off in experiment 2), suggesting that intuitive "fixes" such as placing one's phone face down or turning it off are likely futile. However, our data suggest at least one simple solution: separation. Although this approach may seem at odds with prior research indicating that being separated from one's phone undermines performance by increasing anxiety (Cheever et al. 2014; Clayton et al. 2015), we note that participants in those studies were unexpectedly separated from their phones (Cheever et al. 2014) and forced to hear them ring while being unable to answer (Clayton et al. 2015). In contrast, participants in our experiments expected to be separated from their phones (this was the norm in the lab) and were not confronted with unanswerable notifications or calls while separated. We therefore suggest that defined and protected periods of separation, such as these, may allow consumers to perform better not just by reducing interruptions but also by increasing available cognitive capacity.

Our theoretical framework draws on prior research outlining the role of limited-capacity attentional resources in inhibiting responses to high-priority but task-irrelevant stimuli (Shallice 1972; Bargh 1982; Lavie et al. 2004; Clapp et al. 2009). However, our data are equally consistent with an alternate explanation: that these attentional resources are recruited for purposes of hypervigilance, or monitoring high-priority stimuli in the absence of conscious awareness (e.g., Legrain et al. 2011; Jacob, Jacobs, and Silvanto 2015).
This interpretation is consistent with the common phenomenon of "phantom vibration syndrome," or the feeling that one's phone is vibrating when it actually is not (e.g., Rothberg et al. 2010; Deb 2015). Data suggest that $89 \%$ of mobile phone users experience phantom vibrations at least occasionally (Drouin, Kaiser, and Miller 2012), and that this over-responsiveness to innocuous sensations is particularly prevalent in those whose devices are particularly meaningful (e.g., Rothberg et al. 2010). Because the same limited-capacity attentional resources are implicated in both hypervigilance and inhibition, our data cannot distinguish between the two theoretical explanations. In fact, it is plausible that these processes may operate in tandem, as goal-directed attentional control processes both monitor for signals of potentially important information from highpriority stimuli, and (attempt to) prevent these stimuli from interrupting conscious attention until such signals appear.

\section{Implications and Future Directions}

Consumers' limited cognitive resources shape innumerable aspects of their daily lives, from their approaches to decisions (Bettman et al. 1991) to their enjoyment of experiences (Weber et al. 2009). Our data suggest that the mere presence of consumers' own smartphones may further constrain their already limited cognitive capacity by taxing the attentional resources that reside at the core of both working memory capacity and fluid intelligence. The specific cognitive capacity measures used in our experiments are associated with domain-general capabilities that support fundamental processes such as learning, logical reasoning, abstract thought, problem solving, and creativity (e.g., Cattell 1987; Kane et al. 2004). Because consumers' smartphones are so frequently present, the mere presence effects observed in our experiments have the potential to influence consumer welfare across a wide range of contexts: when consumers work, shop, take classes, watch movies, dine with friends, attend concerts, play games, receive massages, read books, and more (Isikman et al. 2016). Moreover, results from our pilot study (reported prior to experiment 1 ) indicate that the majority of consumers typically keep their smartphones nearby and in sight, where smartphone salience is particularly high.

Consumer Choice. Prior research indicates that occupying cognitive resources by increasing cognitive load causes consumers to rely less on analytic and deliberative "system 2" processing, and more on intuitive and heuristic-based "sys- 
tem 1" approaches (Evans 2008). To the extent that both cognitive load and the mere presence of consumers' smartphones reduce available cognitive capacity, we may expect consumers to be more likely to adopt choice strategies associated with system 1 when their smartphones are present but irrelevant to the choice task. Reliance on system 1 processing could, for example, enhance the appeal of affect-rich choice alternatives (Rottenstreich, Sood, and Brenner 2007), amplify the preference for simple (and possibly inferior) solutions (Drolet, Luce, and Simonson 2009), increase consumers' willingness to make attribute trade-offs (Drolet and Luce 2004), and heighten susceptibility to anchoring effects (Deck and Jahedi 2015). Building on these connections, future research could explore whether the presence of smartphones accentuates individuals' preference for options favored by system 1 processing.

Advertising Effectiveness. The availability of cognitive resources also predicts elaboration likelihood (e.g., Petty and Cacioppo 1986) and susceptibility to deceptive advertising (Xie and Boush 2011). Consistent with a potential shift toward reliance on system 1 processing, consumers who view advertising messages in the presence of their smartphones may be less likely to elaborate on advertising messages and more likely to be influenced by heuristics such as likability of the communicator (e.g., Chaiken 1980). Note that the proposed theoretical framework suggests that this may not be the case for advertising delivered via smartphone, because the cognitive costs associated with mere presence should be incurred when consumers' phones are present but not in use.

Education. Younger adults-92\% of whom are smartphone owners - rely heavily on smartphones (Pew Research Center 2016). Given that many of them are in school, the potential detrimental effects of smartphones on their cognitive functioning may have an outsized effect on long-term welfare. As educational institutions increasingly embrace "connected classrooms" (e.g., Petrina 2007), the presence of students' mobile devices in educational environments may undermine both learning and test performance-particularly when these devices are present but not in use. Future research could focus on how children, adolescents, and young adults are affected by the mere presence of personally relevant technologies in the classroom.

Intentional Disconnection. Although we have primarily focused on the cognitive costs associated with the presence of smartphones, our research is equally relevant to the potential implications of their absence. Discussions of "disconnection" in popular culture reflect increasing consumer interest in intentionally reducing - or at least controlling - the extent to which they interact with their devices (e.g., Perlow 2012; Harmon and Mazmanian 2013). Some consumers are replacing their smartphones with feature phones (i.e., phones lacking the advanced functionality of smartphones; Thomas 2016), others are supplementing their smartphones with stripped-down devices that offer "a short break from connectedness" (http://www.thelightphone.com/), and still others are turning to apps that track, filter, and limit smartphone usage (e.g., https://inthemoment.io/). Our research suggests that these measures may be doubly beneficial for the digitally weary; by redefining the relevance of their devices, these consumers may both reduce digital distraction and increase available cognitive capacity. More broadly, our research contributes to the growing discussion among consumers and marketers alike about the influence of technology on consumers - and consumers on technology - in an increasingly connected world.

One's smartphone is more than just a phone, a camera, or a collection of apps. It is the one thing that connects everything - the hub of the connected world. The presence of one's smartphone enables on-demand access to information, entertainment, social stimulation, and more. However, our research suggests that these benefits - and the dependence they engender - may come at a cognitive cost.

\section{REFERENCES}

Andrews, Sally, David A. Ellis, Heather Shaw, and Lukasz Piwek (2015), "Beyond Self-Report: Tools to Compare Estimated and Real-World Smartphone Use," PLoS One, 10 (10), 1-9.

Baddeley, Alan D. (2003), "Working Memory: Looking Back and Looking Forward," Nature Reviews Neuroscience, 4 (10), 829-39.

Baddeley, Alan D., and Graham Hitch (1974), "Working Memory," Psychologv of Learning and Motivation, 8, 47-89.

Bargh, John A. (1982), "Attention and Automaticity in the Processing of Self-Relevant Information," Journal of Personality and Social Psychology, 43 (3), 425-36.

Baron, Reuben M., and David A. Kenny (1986), “The Moderator-Mediator Variable Distinction in Social Psychological Research: Conceptual, Strategic, and Statistical Considerations," Journal of Personality and Social Psychology, 51 (6), 1173-82.

Benjamin, Daniel, Sebastian Brown, and Jesse Shapiro (2013), "Who Is 'Behavioral'? Cognitive Ability and Anomalous Preferences," Journal of the European Economic Association, 11 (6), 1231-55.

Bettman, James R. (1979), An Information Processing Theory of Consumer Choice, Reading, MA: Addison-Wesley.

Bettman, James R., Eric J. Johnson, and John W. Payne (1991), “Consumer Decision Making," in Handbook of Consumer Behavior, ed. Thomas S. Robertson and Harold H. Kassarjian, Englewood Cliffs, NJ: Prentice Hall. 
Bezdjian, Serena, Laura A. Baker, Dora Isabel Lozano, and Adrian Raine (2009), "Assessing Inattention and Impulsivity in Children during the Go/NoGo Task," British Journal of Developmental Psychology, 27 (2), 365-83.

Bizer, George Y., and Robert M. Schindler (2005), "Direct Evidence of Ending-Digit Drop-off in Price Information Processing," Psychologv and Marketing, 22 (10), 771-83.

Bowman, Laura L., Laura E. Levine, Bradley M. Waite, and Michael Gendron (2010), "Can Students Really Multitask? An Experimental Study of Instant Messaging while Reading," Computers and Education, 54 (4), 927 31.

Caird, Jeff K., Chelsea R. Willness, Piers Steel, and Chip Scialfa (2008), “A Meta-Analysis of the Effects of Cell Phones on Driver Performance," Accident Analysis and Prevention, 40 (4), 1282-93.

Cattell, Raymond B. (1987), Intelligence: Its Structure, Growth and Action, New York: Elsevier.

Chaiken, Shelly (1980), "Heuristic versus Systematic Information Processing and the Use of Source versus Message Cues in Persuasion," Journal of Personality and Social Psychology, 39 (5), 752-66.

Cheever, Nancy A., Larry D. Rosen, L. Mark Carrier, and Amber Chavez (2014), "Out of Sight Is Not Out of Mind: The Impact of Restricting Wireless Mobile Device Use on Anxiety Levels among Low, Moderate and High Users," Computers in Human Behavior, 37 (August), 290-97.

Clapp, Wesley C., and Adam Gazzaley (2012), "Distinct Mechanisms for the Impact of Distraction and Interruption on Working Memory in Aging," Neurobiology of Aging, 33 (1), 134-48.

Clapp, Wesley C., Michael T. Rubens, and Adam Gazzaley (2009), "Mechanisms of Working Memory Disruption by External Interference," $\mathrm{Ce}$ rebral Cortex, 20 (4), 859-72.

Clayton, Russell B., Glenn Leshner, and Anthony Almond (2015), “The Extended iSelf: The Impact of iPhone Separation on Cognition, Emotion, and Physiology," Journal of Computer-Mediated Communication, 20 (2), 119-35.

Corbetta, Maurizio, and Gordon L. Shulman (2002), "Control of GoalDirected and Stimulus-Driven Attention in the Brain," Nature Reviews Neuroscience, 3 (3), 201-15.

Costello, Anna B., and Jason W. Osborne (2005), "Best Practices in Exploratory Factor Analysis: Four Recommendations for Getting the Most from Your Analysis," Practical Assessment, Research, and Evaluation, 10 (7), 1-9.

Craik, Fergus I. M., and Robert S. Lockhart (1972), "Levels of Processing: A Framework for Memory Research," Journal of Verbal Learning and Verbal Behavior, 11 (6), 671-84.

Deb, Amrita (2015), "Phantom Vibration and Phantom Ringing among Mobile Phone Users: A Systematic Review of Literature," Asia-Pacific Psychiatry, 7 (3), 231-39.

Deck, Cary, and Salar Jahedi (2015), "The Effect of Cognitive Load on Economics Decision Making: A Survey and New Experiments," European Economic Review, 78 (C), 97-119.

Deutsche Telekom AG (2012), "Smart Payments-How the Cell Phone Becomes a Wallet," research report, http://www.studie-life.de/en/life -reports/smart-payments/.

Drolet, Aimee, and Mary Frances Luce (2004), "The Rationalizing Effects of Cognitive Load on Emotion-Based Trade-off Avoidance," Journal of Consumer Research, 31 (1), 63-77.

Drolet, Aimee, Mary Frances Luce, and Itamar Simonson (2009), "When Does Choice Reveal Preference? Moderators of Heuristic versus GoalBased Choice," Journal of Consumer Research, 36 (1), 137-47.
Drouin, Michelle, Daren H. Kaiser, and Daniel A. Miller (2012), "Phantom Vibrations among Undergraduates: Prevalence and Associated Psychological Characteristics," Computers in Human Behavior, 28 (4), 1490-96. dscout (2016), "Mobile Touches: dscout's Inaugural Study on Humans and Their Tech," research report, https://blog.dscout.com/hubfs/downloads /dscout_mobile_touches_study_2016.pdf.

End, Christian M., Shaye Worthman, Mary Bridget Mathews, and Katharina Wetterau (2009), "Costly Cell Phones: The Impact of Cell Phone Rings on Academic Performance," Teaching of Psychology, 37 (1), 55-57.

Engle, Randall W. (2002), "Working Memory Capacity as Executive Attention," Current Directions in Psychological Science, 11 (1), 19-23.

Engle, Randall W., Judy Cantor, and Julie J. Carullo (1992), "Individual Differences in Working Memory and Comprehension: A Test of Four Hypotheses," Journal of Experimental Psychology: Learning, Memory, and Cognition, 18 (5), 972-92.

Engle, Randall W., Stephen W. Tuholski, James E. Laughlin, and Andrew R. A. Conway (1999), "Working Memory, Short-Term Memory, and General Fluid Intelligence: A Latent-Variable Approach,” Journal of Experimental Psychologv: General, 128 (3), 309-31.

Evans, Jonathan St. B. T. (2008), "Dual-Processing Accounts of Reasoning, Judgment, and Social Cognition," Annual Review of Psychologv, 59, 25578.

Fecteau, Jillian H., and Douglas P. Munoz (2006), "Salience, Relevance, and Firing: A Priority Map for Target Selection," Trends in Cognitive Sciences, 10 (8), 382-90.

Fox, Annie Beth, Jonathan Rosen, and Mary Crawford (2009), "Distractions, Distractions: Does Instant Messaging Affect College Students' Performance on a Concurrent Reading Comprehension Task?" CuberPsychology and Behavior, 12 (1), 51-53.

Froese, Arnold D., Christina N. Carpenter, Denyse A. Inman, Jessica R. Schooley, Rebecca B. Barnes, Paul W. Brecht, and Jasmin D. Chacon (2012), "Effects of Classroom Cell Phone Use on Expected and Actual Learning," College Student Journal, 46 (2), 323-32.

Geller, Valerie, and Phillip Shaver (1976), "Cognitive Consequences of SelfAwareness," Journal of Experimental Social Psychology, 12 (1), 99-108.

Halford, Graeme S., Nelson Cowan, and Glenda Andrews (2007), "Separating Cognitive Capacity from Knowledge: A New Hypothesis," Trends in Cognitive Sciences, 11 (6), 236-42.

Harmon, Ellie, and Melissa Mazmanian (2013), "Stories of the Smartphone in Everyday Discourse: Conflict, Tension and Instability," in Proceedings of the SIGCHI Conference on Human Factors in Computing Systems, New York: ACM.

Hofmann, Wilhelm, Fritz Strack, and Roland Deutsch (2008), "Free to Buy? Explaining Self-Control and Impulse in Consumer Behavior," Journal of Consumer Psychology, 18 (1), 22-26.

Horn, John L. (1972), "State, Trait and Change Dimensions of Intelligence," British Journal of Educational Psychologv, 42 (2), 159-85.

Ilkowska, Malgorzata, and Randall W. Engle (2010), "Trait and State Differences in Working Memory Capacity," in Handbook of Individual Differences in Cognition: Attention, Memory, and Executive Control, ed. Aleksandra Gruszka, Gerald Matthews, and Błazej Szymura, New York: Springer.

Isikman, Elif, Deborah J. MacInnis, Gülden Ülkümen, and Lisa A. Cavanaugh (2016), "The Effects of Curiosity-Evoking Events on Activity Enjoyment," Journal of Experimental Psychologv: Applied, 22 (3), 319-30.

Jacob, Jane, Christianne Jacobs, and Juha Silvanto (2015), “Attention, Working Memory, and Phenomenal Experience of WM Content: Memory Levels Determined by Different Types of Top-Down Modulation," Frontiers in Psuchologv, 6, 1603. 
Jaeggi, Susanne M., Martin Buschkuehl, John Jonides, and Walter J. Perrig (2008), "Improving Fluid Intelligence with Training on Working Memory," Proceedings of the National Academv of Sciences, 105 (19), 6829-33.

Johnston, William A., and Veronica J. Dark (1986), "Selective Attention," Annual Review of Psychology, 37 (1), 43-75.

Kahneman, Daniel (1973), Attention and Effort, Englewood Cliffs, NJ: Prentice-Hall.

Kane, Michael J., David Z. Hambrick, Stephen W. Tuholski, Oliver Wilhelm, Tabitha W. Payne, and Randall W. Engle (2004), "The Generality of Working Memory Capacity: A Latent-Variable Approach to Verbal and Visuospatial Memory Span and Reasoning," Journal of Experimental Psuchologv: General, 133 (2), 189-217.

Kernan, Jerry B. (1979), "Presidential Address: Consumer Research and the Public Purpose," in Advances in Consumer Research, ed. William L. Wilkie, Ann Arbor, MI: Association for Consumer Research.

Lane, David M. (1982), "Limited Capacity, Attention Allocation, and Productivity," in Human Performance and Productivity: Information Processing Approaches, ed. W. C. Howell and E. A. Fleishman, Hillsdale, NJ: Erlbaum.

Lavie, Nilli, Aleksandra Hirst, Jan W. de Fockert, and Essi Viding (2004), "Load Theory of Selective Attention and Cognitive Control," Journal of Experimental Psychologv: General, 133 (3), 339-54.

Lee, Stephanie (2016), "Quantifying the Benefits of Smartphone Adoption: Digital Device Substitution and Digital Consumption Expansion," Working Paper, Stanford University.

Legrain, Valéry, Gian Domenicao Iannetti, Léon Plaghki, and André Mouraux (2011), "The Pain Matrix Reloaded: A Salience Detection System for the Body," Progress in Neurobiology, 93 (1), 111-24.

Lynch, John G., and Thomas K. Srull (1982), "Memory and Attentional Factors in Consumer Choice: Concepts and Research Methods," Journal of Consumer Research, 9 (1), 18-37.

Mani, Anandi, Sendhil Mullainathan, Eldar Shafir, and Jiaying Zhao (2013), "Poverty Impedes Cognitive Function," Science, 341 (6149), 976-80.

Mick, David G. (2006), "Meaning and Mattering through Transformative Consumer Research," in Advances in Consumer Research, ed. C. Pechmann and L. L. Price, Provo, UT: Association for Consumer Research.

Miyake, Akira, and Priti Shah (1999), Models of Working Memorv: Mechanisms of Active Maintenance and Executive Control, Cambridge: Cambridge University Press.

Moray, Neville (1959), "Attention in Dichotic Listening: Affective Cues and the Influence of Instructions," Quarterlv Journal of Experimental Psvchologv, 11 (1), 56-60.

Moskowitz, Gordon B. (2002), "Preconscious Effects of Temporary Goals on Attention," Journal of Experimental Social Psychology, 38 (4), 397-404.

Newell, Allen, and Herbert A. Simon (1972), Human Problem Solving, Englewood, NJ: Prentice-Hall.

Oulasvirta, Antti, Tye Rattenbury, Lingyi Ma, and Eeva Raita (2011), "Habits Make Smartphone Use More Pervasive," Personal and Ubiquitous Computing, 16 (1), 105-14.

Perlow, Leslie A. (2012), Sleeping with Your Smartphone: How to Break the 24/7 Habit and Change the Way You Work, Boston: Harvard Business Review Press.

Petrina, Stephen (2007), Advanced Teaching Methods for the Technology Classroom, Hershey, PA: Information Science Publishing.

Petty, Richard E., and John T. Cacioppo (1986), Communication and Persuasion: Central and Peripheral Routes to Attitude Change, New York: Springer-Verlag.
Pew Research Center (2015), “U.S. Smartphone Use in 2015,” Report, Pew Research Center, Washington, DC.

(2016), "Smartphone Ownership and Internet Usage Continues to Climb in Emerging Economies," Report, Pew Research Center, Washington, DC.

(2017), “Mobile Fact Sheet, January 12, 2017,” Report, Pew Research Center, Washington, DC

Radwanick, Sarah (2012), "Five Years Later: A Look Back at the Rise of the iPhone," comScore, June 29.

Raven, John, John C. Raven, and John Hugh Court (1998), Manual for Raven's Progressive Matrices and Vocabulary Scales, San Antonio, TX: Harcourt Assessment.

Recarte, Miguel A., and Luis M. Nunes (2003), "Mental Workload while Driving: Effects on Visual Search, Discrimination, and Decision Making," Journal of Experimental Psychology: Applied, 9 (2), 119-37.

Rothberg, Michael B., Ashish Arora, Jodie Hermann, Reva Kleppel, Peter St. Marie, and Paul Visintainer (2010), "Phantom Vibration Syndrome among Medical Staff: A Cross Sectional Survey," British Medical Journal, doi:10.1136/bmj.c6914.

Rottenstreich, Yuval, Sanjay Sood, and Lyle Brenner (2007), "Feeling and Thinking in Memory-Based versus Stimulus-Based Choices," Journal of Consumer Research, 33 (4), 461-69.

Roye, Anja, Thomas Jacobsen, and Erich Schröger (2007), "Personal Significance Is Encoded Automatically by the Human Brain: An EventRelated Potential Study with Ringtones," European Journal of Neuroscience, 26 (3), 784-90.

Sciandra, Michael, and Jeffrey Inman (2016), "Digital Distraction: Consumer Mobile Device Use and Decision Making," https://papers.ssrn .com/sol3/papers2.cfm?abstract_id=2439202.

Shallice, Tim (1972), "Dual Functions of Consciousness," Psychological Review, 79 (5), 383-93.

Shiffrin, Richard M., and Walter Schneider (1977), "Controlled and Automatic Human Information Processing: II. Perceptual Learning, Automatic Attending and a General Theory," Psychological Review, 84 (2), 127-90.

Soto, David, Dietmar Heinke, Glyn W. Humphreys, and Manuel J. Blanco (2005), "Early, Involuntary Top-Down Guidance of Attention from Working Memory," Journal of Experimental Psychology: Human Perception and Performance, 31 (2), 248-61.

Stothart, Cary, Ainsley Mitchum, and Courtney Yehnert (2015), "The Attentional Cost of Receiving a Cell Phone Notification," Journal of Experimental Psychologv: Human Perception and Performance, 41 (4), 893-97.

Strayer, David L., and William A. Johnston (2001), "Driven to Distraction: Dual-Task Studies of Simulated Driving and Conversing on a Cellular Telephone," Psychological Science, 12 (6), 462-66.

Sullivan, Andrew (2016), "I Used to Be a Human Being," New York Magazine, September.

Thomas, Daniel (2016), "Digitally Weary Users Switch to 'Dumb' Phones," Financial Times, February 21.

Thompson-Booth, Chloe, Essi Viding, Linda C. Mayes, Helena J. V. Rutherford, Sara Hodsoll, and Eamon J. McCrory (2014), "Here's Looking at You, Kid: Attention to Infant Emotional Faces in Mothers and Nonmothers," Developmental Science, 17 (1), 35-46.

Thornton, Bill, Alyson Faires, Maija Robbins, and Eric Rollins (2014), “The Mere Presence of a Cell Phone May Be Distracting: Implications for Attention and Task Performance," Social Psychologv, 45 (6), 479-88.

Turkle, Sherry (2011), Alone Together: Why We Expect More from Technology and Less from Ourselves, New York: Simon \& Schuster. 
Turner, Marilyn L., and Randall W. Engle (1989), "Is Working Memory Capacity Task Dependent?” Journal of Memory and Lanquage, 28 (2), 127-54.

Unsworth, Nash, Richard P. Heitz, Josef C. Schrock, and Randall W. Engle (2005), "An Automated Version of the Operation Span Task," Behavior Research Methods, 37 (3), 498-505.

Vogt, Julia, Jan De Houwer, Agnes Moores, Stefaan Van Damme, and Geert Crombez (2010), "The Automatic Orienting of Attention to Goal-Relevant Stimuli," Acta Psychologica, 134 (1), 61-69.

Weber, René, Ron Tamborini, Amber Westcott-Baker, and Benjamin Kantor (2009), "Theorizing Flow and Media Enjoyment as Cognitive Synchro- nization of Attentional and Reward Networks," Communication Theorv, 19 (4), 397-422.

Wingenfeld, Katja, Christoph Mensebach, Martin Driessen, Renate Bullig, Wolfgang Hartje, and Thomas Beblo (2006), "Attention Bias towards Personally Relevant Stimuli: The Individual Emotional Stroop Task," Psuchological Reports, 99 (3), 781-93.

Xie, Guang-Xin, and David M. Boush (2011), "How Susceptible Are Consumers to Deceptive Advertising Claims? A Retrospective Look at the Experimental Research Literature," Marketing Review, 11 (3), 293314. 